\title{
Does a blanking period after pulmonary vein isolation impact long-term results? Results after 55 months of follow-up
}

Piotr Lodziński, Marek Kiliszek, Edward Koźluk, Agnieszka Piątkowska, Paweł Balsam, Janusz Kochanowski, Piotr Scisło, Radosław Piątkowski, Grzegorz Opolski

$1^{\text {st }}$ Chair and Department of Cardiology, Medical University of Warsaw, Warsaw, Poland

\begin{abstract}
Background: The aims of the study are 1) to assess antiarrhythmic prophylaxis efficacy during the first 2 months after radiofrequency ablation $(A R F)$ due to $A F$; 2) to define risk factors for early $A F$ recurrence (EAFR) after $A R F ; 3)$ to determine the long-term follow-up results and risk factors for late $A F$ recurrence (LAFR).
\end{abstract}

Methods: A total number of 210 consecutive patients who had undergone ARF due to AF were analyzed. Patients were randomized into three groups: Group 1 (G1), without any antiarrhythmic drug (AAD); Group 2 (G2), with amiodarone or sotalol; Group 3 (G3), with last ineffective $A A D$. The study was designed to analyze two periods: short-term observation, the first 2 months after ARF; and at least 2 years of long-term follow-up.

Results: After 2 months, clinical data were collected from 171 patients (123 males, mean age of 50.3 years; persistent $A F$ in 19.8\%; lone AF in 36.6\%). Sinus rhythm (SR) was maintained in 84 (49.1\%) patients; 35 (20.4\%) patients presented with a single episode of AF, 39 (23\%) patients experienced a reduction in number of AF episodes, and 13 (7.5\%) patients showed no improvement. No predisposing factor for early recurrence was found. After a mean follow-up of 55 months, clinical data were collected in 137 patients, of which 47 (34\%) maintained SR. Those more likely to sustain SR were: males (82.9\% vs. $62.2 \% ; p=0.018)$, younger patients (44.8 $\pm 12.7 v$ vs. $52.5 \pm 9.9 ; p=0.0001)$, patients with smaller left atrium diameter $(4.05 \pm$ $\pm 0.49 \mathrm{~cm}$ vs. $4.25 \pm 0.51 \mathrm{~cm} ; p=0.04)$, and those without any AF recurrence during the first 2 months after $A R F(78.7 \%$ vs. 35.6\%; $p<0.0001)$. In the multivariable analysis, the independent risk factors for LAFR were hypertension $(p<0.001)$ and persistent $A F(p=0.014)$.

Conclusions: Antiarrhythmic prophylaxis does not affect the number of AF recurrences during the first 2 months after ablation. SR maintenance during a blanking period after AF ablation is a positive prognostic factor in long-term follow-up. Persistent $A F$ and hypertension are independent risk factors for late AF recurrence after pulmonary vein isolation. (Cardiol J 2014; 21, 4: 384-391)

Key words: pulmonary vein isolation, atrial fibrillation, early AF recurrence, late $\mathrm{AF}$ recurrence

Address for correspondence: Dr Piotr Lodziński, $1^{\text {st }}$ Chair and Department of Cardiology, Medical University of Warsaw, ul. Banacha 1a, 02-097 Warszawa, Poland, e-mail: piotr.lodzinski@me.com

Received: 22.06.2013 Accepted: 07.09.2013 


\section{Introduction}

Atrial fibrillation $(\mathrm{AF})$ is the most common supraventricular tachyarrhythmia and is responsible for one third of all hospital admissions due to arrhythmia. The incidence of AF is $1-2 \%$ of the total population and increases with the age $[1,2]$ up to $8 \%$ in the population over 80 years [3].

Radiofrequency ablation with pulmonary vein isolation (PVI) is an accepted method for treatment of AF [4]. Despite the sophisticated technology involved in $\mathrm{AF}$ ablation therapy, however, $\mathrm{AF}$ recurrence may be observed either in the early period after PVI or during long-term follow-up. In some patients, temporal exacerbation of $\mathrm{AF}$ is observed during the early period after PVI. Furthermore, it is not clearly defined when the early periods ends and when the late period starts. Previously, AF recurrences during the first weeks after PVI were treated as a non-significant factor, but nowadays they are regarded to have an impact on long-term PVI failure [5-7].

Antiarrhythmic prophylaxis with limited efficacy may result in adverse effects [8-10]. The role of early $\mathrm{AF}$ recurrence is unclear.

This study has three main aims: 1) to find the risk factors for early and late recurrence of $\mathrm{AF}$ after PVI, 2) to assess the importance of early $\mathrm{AF}$ recurrence, and 3) to check the usefulness of antiarrhythmic prophylaxis during the early period after PVI.

\section{Methods}

The study was conducted in prospective randomized mode. A total number of 210 consecutive patients who had undergone PVI due to AF were analyzed (2003-2007). In all patients, reversible causes of AF were excluded, as was pre-procedural left atrial appendage thrombus. The inclusion criteria were: 1 ) first PVI due to AF; 2) age above 18 years; and 3 ) sinus rhythm (SR) during the first $24 \mathrm{~h}$ after PVI. The exclusion criteria were: 1) reversible cause of AF; 2) bradycardia of $50 \mathrm{bpm}$ or less, atrioventricular or intraventricular blocks; 3) contraindications to antiarrhythmic agents used during study (in case of contraindications to amiodarone, sotalol was used); and 4) PVI procedure with heart tamponade complication.

The study group was randomly divided into three groups:

- Group 1: patients without any antiarrhythmic agent (G1);

- Group 2: patients receiving amiodarone (or sotalol in case of amiodarone contraindications) (G2); and

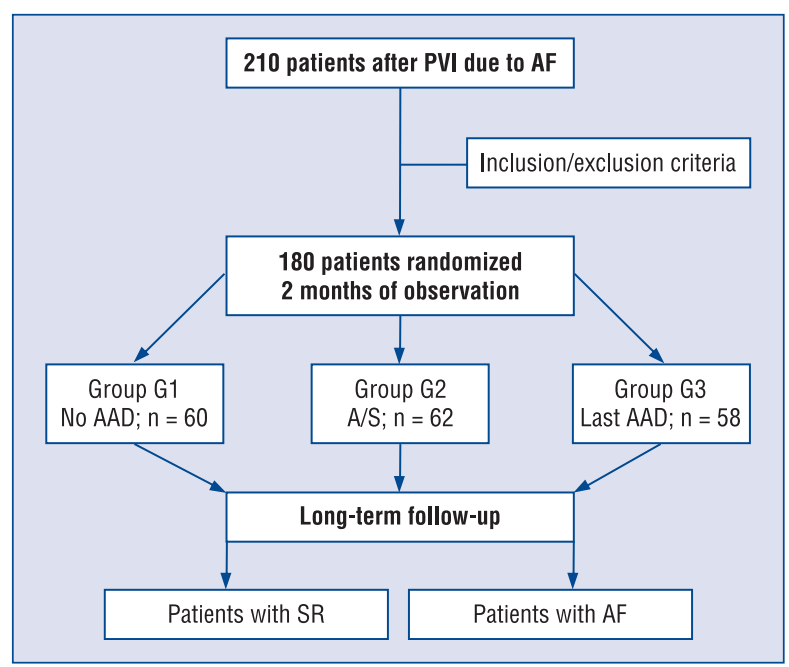

Figure 1. Study flowchart. PVI - pulmonary vein isolation; $\mathrm{AF}$ - atrial fibrillation; $\mathrm{A} / \mathrm{S}$ - amiodarone/sotalol; AAD - antiarrhythmic drug; SR - sinus rhythm.

- Group 3: patients with last ineffective antiarrhythmic agent used before PVI (G3).

The above therapy scheme was continued for 2 months after PVI.

At discharge, all patients were planned for at least two 24-h Holter electrocardiography (ECG) registration, followed by a control visit after the first and second months after PVI. They were also asked to provide symptoms registration during the first 2 months after PVI and to complete ECG documentation in case of any arrhythmia symptoms occurrence. After a minimum period of 2 years after PVI, the patients were called for a follow-up visit, or medical data were obtained through a telephone call. Every episode of AF lasting $30 \mathrm{~s}$ or more was classified as AF recurrence (Fig. 1).

The $\mathrm{AF}$ recurrence rate was assessed after an analysis of all available rhythm recordings: 24-h Holter monitoring at 30 and 60 days after PVI and every ECG registration made on occurrence of symptoms suggesting arrhythmia.

Due to different protocols on result description among different centers, the medical data gathered during follow-up were inconsistent. To make them comparable, the results were divided into groups:

- patients with successful PVI:

- $\quad$ patients without AF recurrence;

- $\quad$ patients with a single AF episode;

- patients with unsuccessful PVI:

- patients with subjective improvement but insignificant reduction in number of AF episodes;

- patients without change in AF intensity. 
During short-term observation, two groups of patients, those without $\mathrm{AF}$ recurrence and those with a single episode of $\mathrm{AF}$, were treated as successful PVI procedures, whereas the groups with a reduction in severity of $\mathrm{AF}$ and without any change were regarded as procedural failures. Analysis was performed separately in the subgroups (no AF, single AF episode, unsuccessful PVI) and the cumulative successful/unsuccessful groups.

During long-term observation, there were only two groups considered: patients with SR and those with documented recurrence of AF. Clinically important, AF recurrence was defined as any documented, ECG-recorded episode of AF lasting at least $30 \mathrm{~s}$.

The study was approved by the local bioethical committee and all patients gave their informed consent.

\section{Antiarrhythmic treatment}

Antiarrhythmic drugs (AAD) were withdrawn 7 days before the procedure in case of amiodarone, beta-blocker, propafenone, and sotalol use, with gradual dosage reduction stopped 2 days before PVI. Antiarrhythmic agents were initiated during the first $24 \mathrm{~h}$ after the procedure.

\section{Pulmonary vein isolation}

In all cases, segmental PVI was done with a $4 \mathrm{~mm}$-tip not irrigated conventional radiofrequency catheter (Marinr, Medtronic) with 30-35 W power and a $50^{\circ} \mathrm{C}$ cutoff ( $\mathrm{RF}$ generator Atakr II, Medtronic). Pulmonary veins were mapped with a 10-pole circular diagnostic catheter with variable diameter (Lasso 2515, Biosense-Webster). LocaLisa system (Medtronic) was used as an additional tool to fluoroscopy for catheter position control during energy delivery.

\section{Study end points}

The end points of the study are: SR maintenance during the first 2 months after PVI, a proarrhythmic event during this period, and SR maintenance during at least 2 years of follow-up.

\section{Statistical analysis}

Continuous data were presented as mean values with standard deviation or as mean values with media. Differences among continuous data were evaluated by Student's t-test. Categorical data were presented as numbers and percentages, and compared with Pearson's $\chi^{2}$ test. The relationship among continuous data was tested with the Spearman rank correlation coefficient. Differences among the three groups were tested with one-way
Table 1. Clinical and demographic characteristic of the study population.

\begin{tabular}{lc}
\hline Age [years] & $50.2 \pm 11.2$ \\
Male & $123(68.3 \%)$ \\
AF duration [months] (med) & $79.2(60)$ \\
Persistent AF & $36(20 \%)$ \\
AF episodes [no/month] (med) & $12.8(8)$ \\
LA diameter [cm] (med) & $4.2(4.2)$ \\
Coronary artery disease & $18(10 \%)$ \\
Hypertension & $83(46.1 \%)$ \\
Diabetes mellitus & $11(6.1 \%)$ \\
Chronic heart failure & $2(1.1 \%)$ \\
Dyslipidemia & $31(17.2 \%)$ \\
Antiarrhythmic drugs & $5 \pm 2$ \\
\hline
\end{tabular}

Continuous variables presented as mean values and standard deviation (SD) or median (med). Categorical variables presented as numbers and percentages; AF - atrial fibrillation; LA — left atrium

ANOVA. Multivariate analysis was performed with the linear regression method. The statistical significance level was $\mathrm{p}<0.05$. Data were analyzed with the STATISTICA (Statsoft Poland) software.

\section{Results}

\section{Two months after ablation}

Table 1 shows the clinical and demographic data of the patients. The study population consisted of 180 patients (mean age of $50.2 \pm 11.2$ years, 123 males). In 36 patients, a persistent form of $\mathrm{AF}$ was recognized.

A total number of 180 patients were randomized into one of the following three groups:

- Group 1 (G1): without antiarrhythmic agent (60 patients);

- Group 2 (G2): with amiodarone (30 patients) or sotalol (32 patients);

- Group 3 (G3): with last ineffective antiarrhythmic agent (58 patients).

Two patients randomized to G3 received sotalol before PVI; therefore, they were moved to G2. Reliable data after 2 months were available in 171 patients (G1: 57 patients; G2: 59 patients; G3: 55 patients). Table 2 presents the groups' characteristics.

\section{Occurrence of end points}

Early period after PVI. During first 2 months after PVI, 84 (49.1\%) patients were free of any AF recurrence, 35 (20.4\%) experienced a single episode of AF, 39 (23\%) showed an insignificant reduction in $\mathrm{AF}$ burden, and $13(7.5 \%)$ presented no change in clinical status. 
Table 2. Characteristics of the study group with short-term follow-up.

\begin{tabular}{lccccc}
\hline & G1 (n= 57) & G2 $(\mathbf{n}=59)$ & G3 (n=55) & Total (n= 171) & P \\
\hline Age [years] & $47.6 \pm 13$ & $50.8 \pm 10$ & $52.5 \pm 11$ & $50.3 \pm 11.2$ & 0.18 \\
Body mass index $\left[\mathrm{kg} / \mathrm{m}^{2}\right]$ & $27.7 \pm 4.7$ & $28.5 \pm 5.1$ & $28.7 \pm 4.6$ & $28.6 \pm 4.9$ & 0.78 \\
LA diameter [cm] & $4.1 \pm 0.4$ & $4.3 \pm 0.6$ & $4.2 \pm 0.6$ & $4.2 \pm 0.6$ & 0.2 \\
Male & $41(71.9 \%)$ & $42(71 \%)$ & $35(63.5 \%)$ & $123(68.3 \%)$ & 0.58 \\
AF duration [months] (med) & $76(48)$ & $80.24(60)$ & $83.3(60)$ & $79.6(60)$ & 0.96 \\
Persistent AF & $8(14 \%)$ & $17(28.8 \%)$ & $9(16.3 \%)$ & $34(19.8 \%)$ & 0.07 \\
AF episodes [month] (med) & $11.5(5.5)$ & $15.5(10)$ & $10.7(8)$ & $12.4(8.5)$ & 0.2 \\
Coronary artery disease & $5(8.8 \%)$ & $7(12 \%)$ & $6(10.9 \%)$ & $18(11 \%)$ & 0.86 \\
Hypertension & $22(39 \%)$ & $29(49 \%)$ & $27(49.1 \%)$ & $78(46 \%)$ & 0.5 \\
Diabetes & $2(3.5 \%)$ & $4(6.8 \%)$ & $4(7.2 \%)$ & $10(5.8 \%)$ & 0.53 \\
Chronic heart failure & $1(1.8 \%)$ & $1(1.6 \%)$ & $0(0 \%)$ & $2(1.2 \%)$ & 0.62 \\
Mitral regurgitation & $1(1.8 \%)$ & $3(5.1 \%)$ & $1(1.8 \%)$ & $5(2.9 \%)$ & 0.24 \\
Dyslipidemia & $8(12 \%)$ & $11(19 \%)$ & $10(18.2 \%)$ & $28(16 \%)$ & 0.82 \\
ACEl & $20(35 \%)$ & $26(44.1)$ & $25(45.5 \%)$ & $71(42 \%)$ & 0.56 \\
Beta-blockers & 0 & 0 & $13(23 \%)$ & $13(7.6 \%)$ & \\
\hline
\end{tabular}

G1 - patients without antiarrhythmic agent; G2 - patients with amiodarone/sotalol; G3 — patients with last ineffective antiarrhythmic agent; ACEI - angiotensin-converting enzyme inhibitor; AF — atrial fibrillation; LA — left atrial

Table 3. End point occurrence in the study group during 2 months of observation.

\begin{tabular}{lccccc}
\hline & G1 (n = 57) & G2 (n=59) & G3 (n= 55) & Total (n= 171) & P \\
\hline AF episodes & & & & \\
Without AF & $31(54.3 \%)$ & $28(47.5 \%)$ & $25(45.5 \%)$ & $84(49.1 \%)$ & 0.34 \\
Single AF episode & $9(15.7 \%)$ & $9(15.2 \%)$ & $17(30.9 \%)$ & $35(20.5 \%)$ & 0.09 \\
Reduction in AF episodes & $13(23 \%)$ & $17(28.8 \%)$ & $9(16.4 \%)$ & $39(23 \%)$ & 0.74 \\
Without change & $4(7 \%)$ & $5(8.5 \%)$ & $4(7.2 \%)$ & $13(7.6 \%)$ & 0.58 \\
Proarrhythmia & & & & $2(1.1 \%)$ \\
OT prolongation & 0 & $2(3.3 \%)$ & 0 & $3(1.7 \%)$ \\
Atypical atrial flutter & 0 & $2(3.3 \%)$ & $1(1.8 \%)$ & $1(0.5 \%)$ \\
AF exacerbation & $1(1.7 \%)$ & 0 & 0 & $6(3.3 \%)$ \\
Total proarrhythmia & $1(1.7 \%)$ & $4(6.6 \%)$ & $1(1.8 \%)$ & \\
\hline
\end{tabular}

P value for G1 vs. G2 vs. G3; abbreviations as in Table 2

Table 3 presents the frequency of particular end points. In Group 2, there was a higher number of persistent $\mathrm{AF}$ patients than in the other groups (G1 vs. G2 vs. G3: $14 \%$ vs. $28.8 \%$ vs. $16.3 \%$; $\mathrm{p}=0.08$ ).

In $\mathrm{G} 1,31$ (54.3\%) patients maintained SR, and $9(15.7 \%)$ patients experienced a single episode of AF. In $40(70 \%)$ patients, the PVI procedure was successful during short-term observation (summary of SR and single AF episode). In the other groups, the results presented were: G2: 28 (47.5\%) patients with SR; 9 (15.2\%) patients with single AF episode - summary successful PVI in 37 (62.7\%) patients, G3, respectively: 25 (45.5\%) patients; $17(30.9 \%)$ patients - summary $42(76.4 \%)$ patients, which was statistically insignificant. No indepen- dent risk factor for early recurrence of $\mathrm{AF}$ was found in the multivariate analysis. More than $90 \%$ of all $\mathrm{AF}$ recurrences occurred during the first 30 days of observation (22 \pm 13.5$)$.

To check for the impact of any antiarrhythmic treatment, the results obtained during the first 2 months after PVI were analyzed for frequency of AF recurrences in two subgroups: G1 and cumulative G2 and G3 (Table 4).

Long-term results. During the mean 55-month observation period, reliable data were available in $137(80.1 \%)$ patients. During this time, 47 patients maintained SR, equivalent to $34 \%$ of the study group. Patients with repetitive PVI were classified under AF recurrence, and the final PVI 
Table 4. Cumulative incidence of atrial fibrillation (AF) in patients without antiarrhythmic treatment and with any antiarrhythmic agent.

\begin{tabular}{lccc}
\hline & Without AAD (n= 57) & On AAD (n = 114) & P \\
\hline Successful PVI (without AF + single episode AF) & $40(70.2 \%)$ & $79(69.3 \%)$ & 0.52 \\
Without AF & $31(54.4 \%)$ & $53(46.5 \%)$ & 0.21 \\
\hline
\end{tabular}

$\mathrm{AAD}$ - antiarrhythmic drug; $\mathrm{PVI}$ — pulmonary vein isolation

Table 5. Characteristics of the study group in long-term observation.

\begin{tabular}{lccc}
\hline & Patients with SR & Patients with AF & P \\
\hline Male gender & $39(82.9 \%)$ & $56(62.2 \%)$ & $\mathbf{0 . 0 1 8}$ \\
Age & $44.8 \pm 12.7$ & $52.5 \pm 9.9$ & $\mathbf{0 . 0 0 0 1}$ \\
Time to recurrence & $176.3 \pm 247.7$ & $249.7 \pm 362.8$ & 0.58 \\
Left atrial diameter [cm] & $4.05 \pm 0.49$ & $4.25 \pm 0.51$ & $\mathbf{0 . 0 4}$ \\
Body mass index $\left[\mathrm{kg} / \mathrm{m}^{2}\right]$ & $28.9 \pm 4.7$ & $28.1 \pm 5.05$ & 0.4 \\
AF duration [months] & $79.95 \pm 73.83$ & $79.73 \pm 55$ & 0.98 \\
Persistent AF & $7(14.8 \%)$ & $20(22.2 \%)$ & 0.21 \\
AF episodes/month & $14.5 \pm 14.3$ & $10.9 \pm 10.4$ & 0.18 \\
Coronary artery disease & $5(10.6 \%)$ & $11 \pm 12.2$ & 0.52 \\
Hypertension & $16(34 \%)$ & $48(53.3 \%)$ & 0.04 \\
Diabetes mellitus & $4(8.5 \%)$ & $5(5.5 \%)$ & 0.38 \\
Dyslipidemia & $6(12.7 \%)$ & $18(20 \%)$ & 0.25 \\
\hline
\end{tabular}

Categorical variables presented as sums and percentages; continuous variables presented as averages and standard deviation (SD). AF duration, persistent $A F$ and number of $A F$ episodes/month - pre-ablation data; $A F$ - atrial fibrillation; SR — sinus rhythm

Table 6. The influence of first 2 months results after pulmonary vein isolation on long-term results.

\begin{tabular}{lcccc}
\hline Results after 2 months & Sinus rhythm & AF recurrence & Long-term success rate & P \\
\hline Without AF & $37(78.7 \%)$ & $32(35.6 \%)$ & $\mathbf{5 3 . 6 \%}$ & $<\mathbf{0 . 0 0 0 1}$ \\
Single AF episode & $5(10.6 \%)$ & $14(15.5 \%)$ & $26.3 \%$ & 0.3 \\
Reduction of AF & $5(10.6 \%)$ & $32(35.5 \%)$ & $15.6 \%$ & 0.0012 \\
No change & $0(0 \%)$ & $12(13.3 \%)$ & $0 \%$ & 0.005 \\
\hline
\end{tabular}

AF - atrial fibrillation

results were not included in further analysis some of repetitive PVI were performed in different center.

There were statistically significant differences among groups with SR and AF recurrence: patients with successful PVI were younger $(44.8 \pm 12.7 \mathrm{vs}$. $52.5 \pm 9.9$ years; $p=0.0001)$, had no hypertension (34\% vs. $53.3 \%$; OR 2.2 ; $95 \%$ CI $1.06-4.6$; $\mathrm{p}=0.04)$, and had a smaller left atrial diameter $(4.05 \pm 0.49 \mathrm{~cm}$ vs. $4.25 \pm 0.55 \mathrm{~cm} ; \mathrm{p}=0.04)$ (Table 5).

The findings obtained from the mean 55-month observation period showed that the antiarrhythmic strategy during the first 2 months of follow-up does not impact long-term results. Among patients with SR during the first 2 months after PVI, 78.7\% had no $\mathrm{AF}$ recurrence during long-term follow-up, compared with $35.6 \%$ in the early recurrence group ( $<<0.0001$; Table 6 ). In summary, the long-term success rate in patients with SR during the first 2 months after PVI was $53.6 \%$, whereas in patients with even one single early recurrence of $\mathrm{AF}$, the percentage dropped to $26.3 \%$ (Fig. 2).

Hypertension and persistent $\mathrm{AF}$ were independent risk factors for $\mathrm{AF}$ recurrence in longterm observation: 0.618 ; $95 \%$ CI $0.395-0.842$; $\mathrm{p}<0.001$ and $0.48 ; 95 \%$ CI $0.103-0.857 ; \mathrm{p}=0.014$, respectively. 


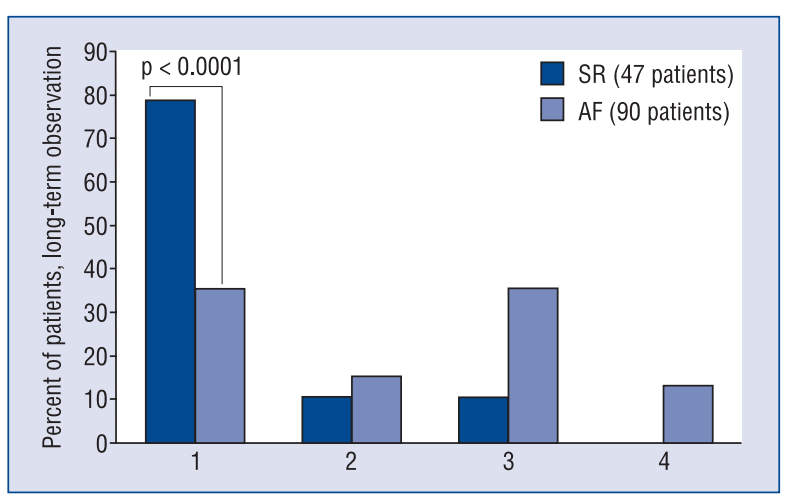

Figure 2. Comparison between long-term and short-term observation results; $\mathrm{SR}$ - sinus rhythm; $\mathrm{AF}$ - atrial fibrillation; Early period results: 1 - no AF; 2 - single $\mathrm{AF}$ episode; 3 - reduction of AF reccurences; $4-$ no change.

\section{Discussion}

This study aimed to verify the hypothesis that AAD impact the results of PVI during the early period. Long-term verification, although difficult, was also performed. Our single-center study group consisted of consecutive patients qualified for PVI, which makes the whole group more representative of the general population.

\section{Influence of post-ablation antiarrhythmic prophylaxis on AF recurrence frequency}

This study found no association between antiarrhythmic strategy and PVI results during the early period after radiofrequency ablation, which is opposite to previously published data [11]. In the 5A study Antiarrhythmics After Ablation of Atrial Fibrillation, Roux at al. [11] assessed antiarrhythmic prophylaxis during the first 6 months after PVI. A total number of 110 patients were randomized into two groups: those using AAD and those without any prophylaxis. The clinical characteristics of the patients presented in this study were similar to those in the $5 \mathrm{~A}$ study population; however, persistent AF patients were excluded in the latter. In the 5A study, AAD resulted in a significant reduction in number of $\mathrm{AF}$ recurrences, hospital admissions, cardioversions, and drug change due to symptomatic atrial arrhythmias: $19 \%$ in the AAD group vs. $42 \%$ in those without AAD $(p=0.005)$. Why do these two similar studies show opposite results? The answer may lie in the study methodology and the assessment criteria for the end result. First of all, the study population was different: in our study, both paroxysmal and per- sistent patients were included, but prior PVI was a criterion for exclusion. Persistent AF patients were distributed among three groups evenly. Thus, the reason may be the difference in the definition of AF recurrence. In both studies, every atrial tachyarrhythmia was treated as AF recurrence, but in the $5 \mathrm{~A}$ study, the time criterion was different from that recommended in the guidelines. That is, the $5 \mathrm{~A}$ study specified that arrhythmia should last at least $24 \mathrm{~h}$ or that the symptoms should lead to earlier cardioversion; according to the guidelines, this time criterion should be $30 \mathrm{~s}$. The definition of recurrence in the 5A study may lead to underestimation of the real number of arrhythmia episodes in a study group. Such a definition seems controversial, especially in the continuation of the 5A study where, during 6 months of observation, $\mathrm{AF}$ recurrence was defined as lasting $60 \mathrm{~s}$ or more [12]. In our study, the AAD group was divided to check the hypothesis that AAD that is ineffective before ablation may be successful in AF prevention after PVI. The results presented in this study showed that this hypothesis is false. To compare the results of both studies, a separate analysis was made on patients without $A A D$ vs. those receiving any AAD. There were no significant differences in recurrence rate in both groups.

\section{AF recurrence risk factors during the early period after PVI}

This study found no risk factor for early AF recurrence (EAFR) after PVI. Other studies obtained similar results, even with the definition of early period extended to 3 months and ECG registration with loop recorders [13].

More than $90 \%$ of all EAFR occurred within the first 30 days after PVI (mean of 22 days). These data are also consistent with those previously published: 54\% EAFR during the first 2 weeks after PVI, $38 \%$ occurring during the next 2 weeks, and at least one clinically significant $\mathrm{AF}$ episode in $65 \%$ of patients [13]. In case of any symptoms suggesting arrhythmia recurrence, a standard ECG was performed. Hindricks et al. [14] screened patients before and after PVI. They reported that before PVI, only symptomatic AF episodes occurred in $38 \%$ of patients, both symptomatic and asymptomatic ones in $57 \%$, and only asymptomatic arrhythmia in 5\%. During 6 months of observation after PVI, only asymptomatic AF was registered in $37 \%$ of patients. Asymptomatic AF recurrences are not only a matter of success assessment. In case of highly symptomatic AF before PVI, patients with asymptomatic arrhythmia after ablation consider 
themselves cured. Improvement of quality of life is one of the therapeutic goals, but asymptomatic AF episodes imply a thromboembolic risk despite patients' better clinical status.

\section{Late $\mathrm{AF}$ recurrence risk factors}

During the mean 55-month observation period, only $34 \%$ of patients maintained SR after a single PVI procedure. The observation period in different publications ranges from 6 months to 6 years. Although single and repetitive procedures were included in these studies, the common conclusion is that long-term results are less optimistic than short-term ones. One study reported a 6 -year success rate of $23 \%$ after a single procedure and of $39 \%$ after repetitive ablation.

In this study, SR maintenance during the first 2 months decreased the late AF recurrence rate significantly independently from prior antiarrhythmic strategy: $78.7 \%$ of patients without late $\mathrm{AF}$ recurrence maintained SR during the first 2 months of observation ( $p<0.0001$ ), and the long-term success rate in this subgroup was $53.6 \%$. Even a single AF episode during the first 2 months reduced this result twice. The available publications during the patient enrolment phase showed no association between early AF recurrence and long-term results [7]. This is opposite to the data presented by Arya et al. [16] and Cai et al. [6]. In the first study, the independent risk factors for late $\mathrm{AF}$ recurrence were: early $\mathrm{AF}$ recurrence (HR 4.3; 95\% CI 2.0-9.1; $p=0.0001)$, left atrial diameter $\geq 50 \mathrm{~mm}$ (HR 4.6; 95\% CI 2.9-9.1; $\mathrm{p}=0.0001$ ), and hypertension (HR 3.3; 95\% CI 1.4-7.5; $\mathrm{p}=0.003)$; in the second, the risk factors were overweight (HR 4.71; 95\% CI 1.71-12.98; $\mathrm{p}=0.003)$, metabolic syndrome (HR $4.41 ; 95 \% \mathrm{CI}$ $1.56-12.46 ; \mathrm{p}=0.005)$, and early AF recurrence (HR 3.18; 95\% CI 1.07-9.44; $p=0.037$ ). In this study, we found no connection between body mass index and increased number of $\mathrm{AF}$ recurrence.

In the Bordeaux group, the 5-year follow-up was based on 24-h Holter registration and standard ECG in case of symptoms suggesting arrhythmia recurrence with duration of $30 \mathrm{~s}$ of more as a diagnostic criterion for a clinically significant episode [15]. In the success rate analysis, a time-dependent reduction in freedom from $\mathrm{AF}$ was observed. After 1,2 , and 5 years of observation, SR was maintained in $40 \%, 37 \%$, and $29 \%$ of patients, respectively. This study group included long-lasting persistent AF patients. This form of arrhythmia was the only independent risk factor for late AF recurrence.

\section{Limitations of the study}

Similar to other studies, the SR documentation method is the most important limitation of this work. The use of 24-h ECG registration and standard ECG in case of symptoms suggesting arrhythmia recurrence may lead to underestimation of the real recurrence rate. The study group consisted of patients from different regions of the country; thus, the results of the Holter ECG monitoring were presented in different manners. To make them comparable, categories of results were used instead of objective arrhythmia counts. Different types of AF may have influence the results although no long lasting persistent $\mathrm{AF}$ patients were enrolled.

\section{Conclusions}

Antiarrhythmic drugs do not reduce the incidence of early AF recurrence after pulmonary vein isolation. SR maintenance during the blanking period seems to be a strong positive predictor of long-term success after ablation. Hypertension and persistent forms of $\mathrm{AF}$ are independent risk factors for late $\mathrm{AF}$ recurrence after PVI.

\section{Conflict of interest: none declared}

\section{References}

1. Go AS, Hylek EM, Phillips KA et al. Prevalence of diagnosed atrial fibrillation in adults: national implications for rhythm management and stroke prevention: The AnTicoagulation and Risk Factors in Atrial Fibrillation (ATRIA) Study. JAMA, 2001; 285: 2370-2375.

2. Camm AJ, Kirchhof P, Lip GY et al. Guidelines for the management of atrial fibrillation: The Task Force for the Management of Atrial Fibrillation of the European Society of Cardiology (ESC). Europace, 2010; 12: 1360-1420.

3. Furberg CD, Psaty BM, Manolio TA, Gardin JM, Smith VE, Rautaharju PM. Prevalence of atrial fibrillation in elderly subjects (the Cardiovascular Health Study). The Am J Cardiol, 1994; 74: 236-241.

4. Camm AJ, Lip GY, De Caterina R et al. 2012 focused update of the ESC Guidelines for the management of atrial fibrillation: An update of the 2010 ESC Guidelines for the management of atrial fibrillation: Developed with the special contribution of the European Heart Rhythm Association. Europace, 2012; 14: 1385-1413.

5. Bertaglia E, Stabile G, Senatore G et al. Predictive value of early atrial tachyarrhythmias recurrence after circumferential anatomical pulmonary vein ablation. Pacing and clinical electrophysiology. PACE, 2005; 28: 366-371.

6. Cai L, Yin Y, Ling $Z$ et al. Predictors of late recurrence of atrial fibrillation after catheter ablation. Int J Cardiol, 2013; 164: 82-87. 
7. O’Donnell D, Furniss SS, Dunuwille A, Bourke JP. Delayed cure despite early recurrence after pulmonary vein isolation for atrial fibrillation. Am J Cardiol, 2003; 91: 83-85.

8. Lafuente-Lafuente C, Mouly S, Longas-Tejero MA, Mahe I, Bergmann JF. Antiarrhythmic drugs for maintaining sinus rhythm after cardioversion of atrial fibrillation: A systematic review of randomized controlled trials. Arch Internal Med, 2006; 166: 719-728.

9. Hohnloser SH, Singh BN. Proarrhythmia with class III antiarrhythmic drugs: Definition, electrophysiologic mechanisms, incidence, predisposing factors, and clinical implications. J Cardiovasc Electrophysiol, 1995; 6 (10 Part 2): 920-936.

10. Friedman PL, Stevenson WG. Proarrhythmia. Am J Cardiol, 1998; 82: 50N-58N.

11. Roux JF, Zado E, Callans DJ et al. Antiarrhythmics After Ablation of Atrial Fibrillation (5A Study). Circulation, 2009; 120: 1036-1040.
12. Leong-Sit P, Roux JF, Zado E et al. Antiarrhythmics after ablation of atrial fibrillation (5A Study): Six-month follow-up study. Circulation, 2011; 4: 11-14.

13. Joshi S, Choi AD, Kamath GS et al. Prevalence, predictors, and prognosis of atrial fibrillation early after pulmonary vein isolation: Findings from 3 months of continuous automatic ECG loop recordings. J Cardiovasc Electrophysiol, 2009; 20: 1089-1094.

14. Hindricks G, Piorkowski C, Tanner H et al. Perception of atrial fibrillation before and after radiofrequency catheter ablation: Relevance of asymptomatic arrhythmia recurrence. Circulation, 2005; 112: 307-313.

15. Weerasooriya R, Khairy P, Litalien J et al. Catheter ablation for atrial fibrillation: Are results maintained at 5 years of follow-up? J Am Coll Cardiol, 2011; 57: 160-166.

16. Arya A, Hindricks G, Sommer P et al. Long-term results and the predictors of outcome of catheter ablation of atrial fibrillation using steerable sheath catheter navigation after single procedure in 674 patients. Europace, 2010; 12: 173-180. 